\title{
A REMARKABLE IDENTITY FOUND IN RAMANUJAN'S THIRD NOTEBOOK
}

\author{
by BRUCE C. BERNDT $\dagger$ and S. BHARGAVA $\ddagger$
}

(Received 14 May, 1991)

1. Introduction. Ramanujan's short third notebook contains 33 pages of unorganized material. On page 386 of the third notebook, in the pagination of the Tata Institute's publication [3], Ramanujan offers the following remarkable identity.

THeOREM 1. Let $a, b, c$, and $d$ be any numbers such that

Then

$$
a d=b c \text {. }
$$

$$
\begin{aligned}
& 64\left\{(a+b+c)^{6}+(b+c+d)^{6}-(c+d+a)^{6}-(d+a+b)^{6}+(a-d)^{6}-(b-c)^{6}\right\} \\
& \times\left\{(a+b+c)^{10}+(b+c+d)^{10}-(c+d+a)^{10}-(d+a+b)^{10}+(a-d)^{10}-(b-c)^{10}\right\} \\
& =45\left\{(a+b+c)^{8}+(b+c+d)^{8}-(c+d+a)^{8}-(d+a+b)^{8}+(a-d)^{8}-(b-c)^{8}\right\}^{2} .
\end{aligned}
$$

The hypothesis (1) was omitted by Ramanujan, although it does appear on page 385 as a hypothesis for some similar, simpler identities, which we will discuss later. If this assumption is removed, a numerical counterexample can be found by simply setting $a=d=1$ and $b=c=0$. Then the left and right sides of (2) are, respectively, $16,498,944$, and $11,704,500$.

We first proved (2) by verifying it with the symbolic algebra system Mathematica. Of course, such a proof is less than satisfactory, for it provides no information on how (2) was discovered or why it exists. We still do not know what paths led Ramanujan to (2). Nonetheless, the purpose of this paper is to give an elementary, algebraic proof of (2) which certainly sheds more light into its raison d'etre than a computer algebra proof.

To prepare for our proof of (2), we reformulate Theorem 1 . Set, for each positive integer $m$,

$$
\begin{aligned}
F_{2 m}(a, b, c, d)=(a+b+c)^{2 m}+(b+c+d)^{2 m}- & (c+d+a)^{2 m} \\
& -(d+a+b)^{2 m}+(a-d)^{2 m}-(b-c)^{2 m} .
\end{aligned}
$$

If we put $b=a x, c=a y$, and $d=a x y$, the hypothesis (1) remains valid. It is then easily seen that

$$
F_{2 m}(a, b, c, d)=a^{2 m} f_{2 m}(x, y)
$$

where

$$
\begin{aligned}
f_{2 m}(x, y)=(1+x+y)^{2 m}+(x+y+x y)^{2 m}- & (y+x y+1)^{2 m} \\
& -(x y+1+x)^{2 m}+(1-x y)^{2 m}-(x-y)^{2 m} .
\end{aligned}
$$

Hence, (2) takes the form

$$
64 f_{6}(x, y) f_{10}(x, y)=45 f_{8}^{2}(x, y) .
$$

$\dagger$ Research partially supported by NSF grant DMS 88-20680.

$\ddagger$ Research supported by a Fulbright fellowship.

Glasgow Math. J. 34 (1992) 341-345. 
From (3), we can easily see by inspection that $x=0,1,-1,-2,-\frac{1}{2}$ are zeros of $f_{2 m}(x, y)$. (By symmetry, $y=0,1,-1,-2,-\frac{1}{2}$ are also zeros.) Since $f_{2 m}$ is of degree (at most) $2 m$ in each of the variables $x$ and $y$, it follows that $f_{2}(x, y) \equiv 0 \equiv f_{4}(x, y)$. Thus, in our earlier notation,

$$
(a+b+c)^{n}+(b+c+d)^{n}+(a-d)^{n}=(c+d+a)^{n}+(d+a+b)^{n}+(b-c)^{n},
$$

for $n=2$ or 4 . The identity (5) then provides a formula for producing two equal sums of three squares or two equal sums of three biquadrates. Despite its simplicity, we have been unable to find (5) in the literature.

Dickson [2, pp. 653-655] cites several formulas providing solutions to

$$
u^{4}+v^{4}+w^{4}=x^{4}+y^{4}+z^{4} .
$$

Ramanujan [3, p. 386] also gives a family of solutions of (6) involving one parameter, i.e.,

$$
3^{4}+\left(2 x^{4}-1\right)^{4}+\left(4 x^{5}+x\right)^{4}=\left(4 x^{4}+1\right)^{4}+\left(6 x^{4}-3\right)^{4}+\left(4 x^{5}-5 x\right)^{4}
$$

For further work of Ramanujan on this and related topics, see Berndt's book [1].

Returning to (3), we see that $f_{2 m}(x, y)$ has degree $2 m-1$ in either $x$ or $y$ when $m \geq 3$. Thus, in particular, we have shown that, for some constant $c$,

$$
f_{6}(x, y)=c x\left(x^{2}-1\right)(x+2)(2 x+1) y\left(y^{2}-1\right)(y+2)(2 y+1) .
$$

Furthermore, it also follows that $f_{6}(x, y) \mid f_{2 m}(x, y)$ for every integer $m \geq 3$. In particular, $f_{6}(x, y) \mid f_{8}(x, y)$, which accounts for one feature of (4). Using Mathematica, we attempted to find further factorizations for $m \leq 10$ of the elegance of (4), but we found none.

2. An ancillary lemma. For convenience, set

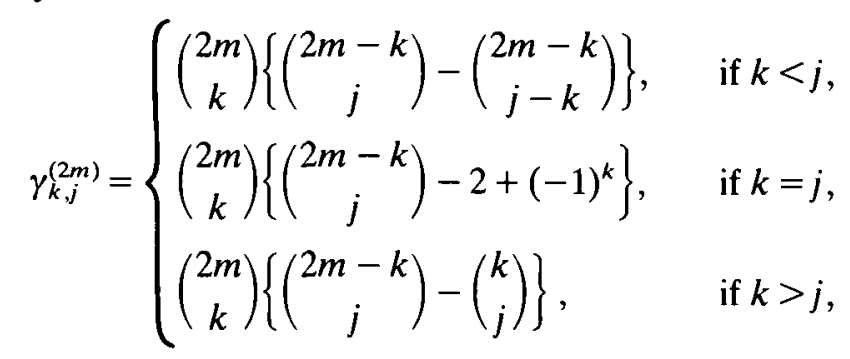

where $1 \leq j, k \leq m-1$. For brevity, we shall often delete the superscript $(2 m)$ in the sequel.

LEMMA 2. Let $\gamma_{j, k}^{(2 m)}$ be defined by (7). Suppose that there exist numbers $\xi_{j}^{(2 m)}, \xi_{k}^{(2 m)}$, and $A_{2 m}$ such that

$$
\gamma_{j, k}^{(2 m)}=A_{2 m} \xi_{j}^{(2 m)} \xi_{k}^{(2 m)}, \quad 1 \leq j, k \leq m-1 .
$$

Then

$$
f_{2 m}(x, y)=A_{2 m} x\left(1-x^{2}\right) H_{2 m}(x) y\left(1-y^{2}\right) H_{2 m}(y),
$$

where, with the superscripts $(2 m)$ suppressed,

$$
H_{2 m}(x)=\sum_{k=1}^{m-1} \xi_{k} x^{k-1}\left(1+x^{2}+\ldots+x^{2 m-2 k-2}\right)
$$


Furthermore, for $m \geq 3$,

$$
H_{2 m}(x)=\xi_{1} \prod_{j=1}^{m-2}\left(1+x^{2}+\rho_{j} x\right),
$$

for certain constants $\rho_{1}, \rho_{2}, \ldots, \rho_{m-2}$, depending upon $m$.

Proof. By (3) and the binomial theorem,

$$
f_{2 m}(x, y)=\sum_{k=0}^{2 m}\left(\begin{array}{c}
2 m \\
k
\end{array}\right) \alpha_{k}(y) x^{k}, \quad m \geq 1,
$$

where

$$
\begin{aligned}
\alpha_{k}(y):=\alpha_{k}^{(2 m)}(y):= & (1+y)^{2 m-k}+(1+y)^{k} y^{2 m-k}-y^{k}(1+y)^{2 m-k} \\
& -(1+y)^{k}+(-y)^{k}-(-1)^{k} y^{2 m-k} .
\end{aligned}
$$

It follows immediately from (13) that

$$
\begin{gathered}
\alpha_{0}(y)=\alpha_{m}(y)=\alpha_{2 m}(y)=0, \\
\alpha_{k}(y)=-\alpha_{2 m-k}(y), \quad 1 \leq k \leq 2 m-1,
\end{gathered}
$$

and

$$
\alpha_{k}(y)=-y^{2 m} \alpha_{k}(1 / y), \quad 1 \leq k \leq 2 m-1 .
$$

From (14), it follows immediately that $f_{2}(x, y) \equiv 0$, as we observed earlier. From (14) and (15), we see that (12) can now be written in the form, for $m \geq 2$,

$$
f_{2 m}(x, y)=\sum_{k=1}^{m-1}\left(\begin{array}{c}
2 m \\
k
\end{array}\right) \alpha_{k}(y)\left(x^{k}-x^{2 m-k}\right) .
$$

We assume hereafter that $m \geq 2$.

Returning to (13), we set

$$
\alpha_{k}(y)=\sum_{j=0}^{2 m} \beta_{k, j}^{(2 m)} y^{j} .
$$

Suppressing the superscripts, we find that, by (13),

$$
\beta_{k, 0}=0, \quad 1 \leq k \leq m-1,
$$

and, from (16), we see that

$$
\beta_{k, j}=-\beta_{k, 2 m-j}, \quad 1 \leq k \leq m-1, \quad 0 \leq j \leq 2 m .
$$

The relations (19) and (20) further imply that

$$
\beta_{k, m}=\beta_{k, 2 m}=0, \quad 1 \leq k \leq m-1 .
$$

Utilizing (19)-(21) in (18), we deduce that

$$
\alpha_{k}(y)=\sum_{j=1}^{m-1} \beta_{k, j}\left(y^{j}-y^{2 m-j}\right), \quad m \geq 2 .
$$


Comparing coefficients of $y^{j}$ in (13) and (22), we find that, for $1 \leq j, k \leq m-1$,

$$
\beta_{k, j}= \begin{cases}\left(\begin{array}{c}
2 m-k \\
j
\end{array}\right)-\left(\begin{array}{c}
2 m-k \\
j-k
\end{array}\right), & \text { if } j>k, \\
\left(\begin{array}{c}
2 m-k \\
j
\end{array}\right)-2+(-1)^{j}, & \text { if } j=k, \\
\left(\begin{array}{c}
2 m-k \\
j
\end{array}\right)-\left(\begin{array}{c}
k \\
j
\end{array}\right), & \text { if } j<k .\end{cases}
$$

Utilizing (23) in (22), then putting (22) in (17), and finally recalling (7), we deduce that

$$
f_{2 m}(x, y)=\sum_{k=1}^{m-1} \sum_{j=1}^{m-1} \gamma_{k, j}\left(x^{k}-x^{2 m-k}\right)\left(y^{j}-y^{2 m-j}\right) \text {. }
$$

Using the assumption (8) in (24), we establish the factorization (9), with $H_{2 m}(x)$ given by (10). From (24), we also see that if $x_{0} \neq 0$ is a zero of $f_{2 m}(x, y)$, then $1 / x_{0}$ is also a zero. Hence, the factorization (11) follows, and so the proof is complete.

By (3), $f_{2 m}(x, y)=f_{2 m}(y, x)$. Hence, from (24), it follows that $\gamma_{k, j}=\gamma_{j, k}, 1 \leq j, k \leq$ $m-1$. Of course, this fact may also readily be verified from (7).

By (7), $\gamma_{1,1}^{(4)}=0$. Hence, from (24), $f_{4}(x, y) \equiv 0$, which we observed earlier.

In fact, the factorization (11) is not really necessary for the proof of Theorem 1.

3. Completion. As noted in the introduction, Theorem 1 is equivalent to (4).

Proof of (4). In turn, we set $m=3,4$, and 5 and calculate the coefficients $\gamma_{k, j}$ from (7). In each case, we find that the condition (8) is satisfied. More specifically, suppressing the superscripts, we find that

$$
\begin{gathered}
A_{6}=3, \quad \xi_{1}=2, \quad \xi_{2}=5 ; \\
A_{8}=8, \quad \xi_{1}=2, \quad \xi_{2}=\xi_{3}=7 ;
\end{gathered}
$$

and

$$
A_{10}=15, \quad \xi_{1}=2, \quad \xi_{2}=9, \quad \xi_{3}=16, \quad \xi_{4}=14 .
$$

Calculating the functions $H_{6}, H_{8}$, and $H_{10}$ by means of (10), we find that

$$
\begin{aligned}
H_{6}(x) & =2\left(1+x^{2}\right)+5 x=(x+2)(2 x+1), \\
H_{8}(x) & =2\left(1+x^{2}+x^{4}\right)+7 x\left(1+x^{2}\right)+7 x^{2} \\
& =2\left(1+x^{2}\right)^{2}+7 x\left(1+x^{2}\right)+5 x^{2}=\left\{2\left(1+x^{2}\right)+5 x\right\}\left(\left(1+x^{2}\right)+x\right) \\
& =(x+2)(2 x+1)\left(x^{2}+x+1\right),
\end{aligned}
$$

and

$$
\begin{aligned}
H_{10}(x) & =2\left(1+x^{2}+x^{4}+x^{6}\right)+9 x\left(1+x^{2}+x^{4}\right)+16 x^{2}\left(1+x^{2}\right)+14 x^{3} \\
& =2\left\{\left(1+x^{2}\right)^{3}-2 x^{2}\left(1+x^{2}\right)\right\}+9 x\left\{\left(1+x^{2}\right)^{2}-x^{2}\right\}+16 x^{2}\left(1+x^{2}\right)+14 x^{3} \\
& =2\left(1+x^{2}\right)^{3}+9 x\left(1+x^{2}\right)^{2}+12 x^{2}\left(1+x^{2}\right)+5 x^{3} \\
& =\left\{2\left(1+x^{2}\right)+5 x\right\}\left\{\left(1+x^{2}\right)+x\right\}^{2} \\
& =(x+2)(2 x+1)\left(x^{2}+x+1\right)^{2} .
\end{aligned}
$$


Hence, from (9), we deduce that

$$
\begin{gathered}
f_{6}(x, y)=3 x\left(1-x^{2}\right)(x+2)(2 x+1) y\left(1-y^{2}\right)(y+2)(2 y+1), \\
f_{8}(x, y)=8 x\left(1-x^{2}\right)(x+2)(2 x+1)\left(x^{2}+x+1\right) y\left(1-y^{2}\right)(y+2)(2 y+1)\left(y^{2}+y+1\right),
\end{gathered}
$$

and

$$
f_{10}(x, y)=15 x\left(1-x^{2}\right)(x+2)(2 x+1)\left(x^{2}+x+1\right)^{2} y\left(1-y^{2}\right)(y+2)(2 y+1)\left(y^{2}+y+1\right)^{2} \text {. }
$$

The elegant identity (4) now easily follows.

\section{REFERENCES}

1. B. C. Berndt, Ramanujan's Notebooks, Part IV, Springer-Verlag, New York, to appear.

2. L. E. Dickson, History of the Theory of Numbers, vol. II, Chelsea, New York, 1952.

3. S. Ramanujan, Notebooks, Vol. 2, Tata Institute of Fundamental Research, Bombay, 1957.

Department of Mathematics

UNIVERSITY OF ILLINOIS

1409 W. GREEN ST.

URBANA,

ILLINOIS 61801

U.S.A.
Department of Mathematics

UNIVERSITY OF MYSORE

MANASA Gangotri

MYSORE 570006

INDIA 